\title{
Modal Analysis of Bracket Structure in Automatic Drill-riveting System Based on ABAQUS
}

\author{
Li Cheng $^{1, a}$, Wang Zhong-qi, ${ }^{1, b}$, Kang Yong-gang ${ }^{1, c}$ and Yang Yuan ${ }^{1, d}$ \\ ${ }^{1}$ The Key Laboratory of Contemporary Design and Integrated Manufacturing Technology, \\ Northwestern Polytechnical University, 710072, Xi'an, Shaanxi, China \\ alicheng2014@mail.nwpu.edu.cn, 'bangzhqi@nwpu.edu.cn, 'kangyonggang@nwpu.edu.cn, \\ dyangyuan0824@mail.nwpu.edu.cn
}

Keywords: Modal analysis, Finite element model, Automatic drill-riveting system, Bracket structure.

\begin{abstract}
The vibration characteristics of bracket structure in automatic drill-riveting system have great effects on the final quality of aircraft panel. Modal analysis can be used to determine the vibration characteristics of mechanical system. After briefly expounding the basic theory of modal analysis, a reasonable finite element model is established and the modal analysis is carried out under the environment of ABAQUS. The result shows that this method for evaluating vibration characteristics of the bracket structure works, can meet the requirements of structure design.
\end{abstract}

\section{Introduction}

Automatic drill-riveting technology is widely used in the assembly of aircraft wing and fuselage panels for its advantages of good drill-riveting quality, high production efficiency, good working condition and easily controlled operations [1]. The bracket is an important part of the automatic drill-riveting system for holding, clamping, moving and locating the aircraft panel. However, during drilling and riveting process, the bracket deformation can lead the riveting panel to over-tolerance due to the large weight and big span length, and degrade the final quality of the panel. So improving vibration resistance of bracket has an important role to guarantee riveting quality.

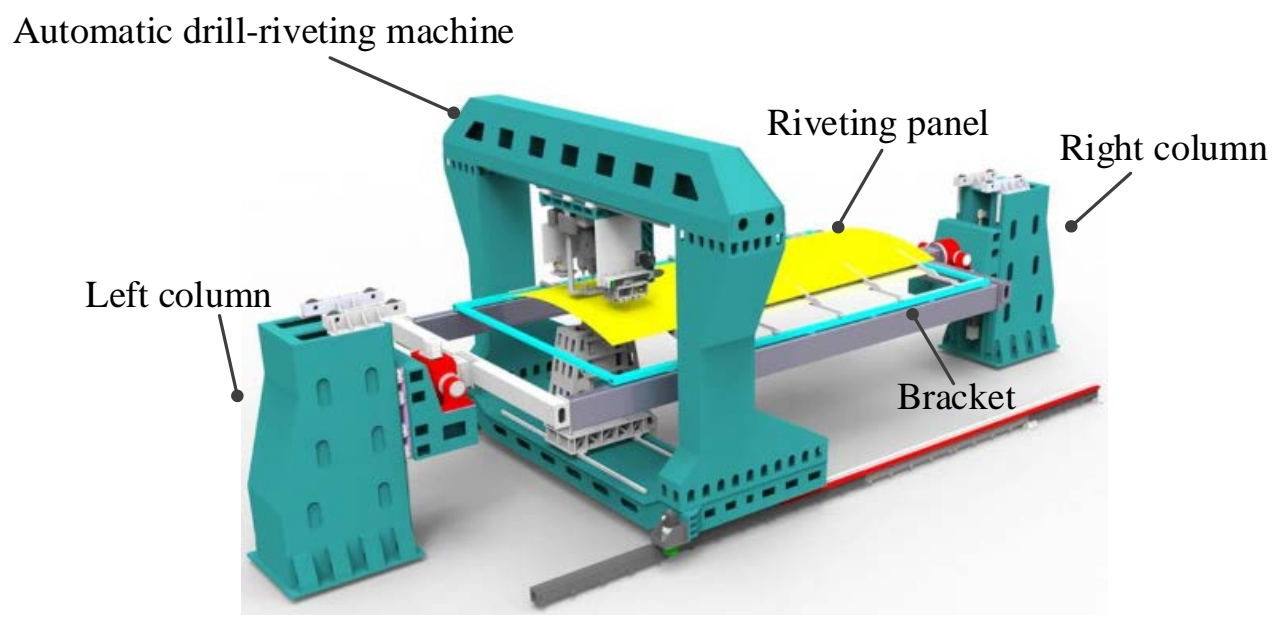

Fig. 1 Automatic drill-riveting system

Modal analysis can be used to determine the vibration characteristics of the mechanical structure, the result of it can explain not only the mechanical stiffness of the structure, but also for the better structure design to get stronger vibration resistance [2]. It has been widely used in machinery, construction, aerospace and chemical industry during last decades [3-6]. In this paper, the finite element dynamic model for the bracket structure in the automatic drill-riveting system is 
built, by using ABAQUS software for modal analysis, the natural frequencies and modal shapes of the bracket and the corresponding stress and deformation can be obtained.

\section{The theory of modal analysis}

Modal analysis is the study of the dynamic properties of structures under vibrational excitation. Modal analysis results can be used to evaluate the dynamic characteristics of the structure, estimate the structural strength, stiffness, stress and strain of products and other structural dynamic vibration problems, it addresses the problems of structural dynamics analysis that other methods are difficult to solve. It is necessary to take a brief analysis of its theory. For a linear time-invariant system with $\mathrm{N}$ degrees of freedom, its motion differential equation is:

$$
M \ddot{X}+C \dot{X}+K X=F X
$$

In the above formula, $\mathrm{M}, \mathrm{C}, \mathrm{K}$ respectively represents the mass matrix, damping matrix and stiffness matrix of the system, $\mathrm{X}$ is the response displacement vector of points, $\mathrm{F}$ is the excitation force vector. Take the formula (1) into the Laplace transform we can get:

$$
\left(s^{2} M+s C+K\right) X(s)=F(s)
$$

Its frequency response function matrix is:

$$
H(w)=\left(K-w^{2} M+j w C\right)^{-1}
$$

Thus, the motion equations of the system can be concluded, $w$ is the system's inherent frequency.

$$
\left(K-w^{2} M+j w C\right) X(w)=F(w)
$$

Set system's degrees of freedom as $\mathrm{N}$, damping type as proportional damping, replace the physical coordinates of the formula with modal coordinates, then we get the r-th order modal:

$$
\left(K_{r}-w^{2} M_{r}+j w C_{r}\right) q_{r}=F_{r}
$$

So the modal coordinates of r-th order modal is:

$$
q_{r}=\frac{F_{r}}{\left(K_{r}-w^{2} M_{r}+j w C_{r}\right)}
$$

From the vibration theory, we know that when the system is a linear time-invariant system, each modal response for a certain point of the system can be obtained by a linear combination [6], then the response of any measuring point is as follows:

$$
x_{l}(w)=\sum_{r=1}^{n} \varphi_{l r} q_{r}=\sum_{r=1}^{n} \frac{\varphi_{l r} F_{r}}{\left(K_{r}-w^{2} M_{r}+j w C_{r}\right)}
$$

In the above formula, for point $l, \varphi_{l r}$ is the modal shape coefficient of the r-th order, let the system a single point of excitation, and the excitation force is applied to the point $p$, then the modal force is:

$$
F_{r}=\varphi_{p r} f_{p}(w)
$$

Based on the above analysis, the frequency response function between response point $\boldsymbol{l}$ and exciting 
point $p$ can be deduced:

$$
\frac{x_{l}(w)}{f_{p}(w)}=H_{l p}(w)=\sum_{r=1}^{n} \frac{\varphi_{l r} \varphi_{p r}}{\left(K_{r}-w^{2} M_{r}+j w C_{r}\right)}
$$

\section{The model building of bracket based on ABAQUS}

In order to reduce the scale of finite element analysis and shorten the computing time, the structure of the bracket and panel have been properly simplified and modified in CATIA V5 [8]. To this end, some specific modified principles are made as follows: (1) ignore the local structures which have little effect on the result or under tiny load, like bolt holes, the excess connection plates, the tensioning straps and so on; (2) reconstruct the frame section as a whole part, without considering the complex contact effects.

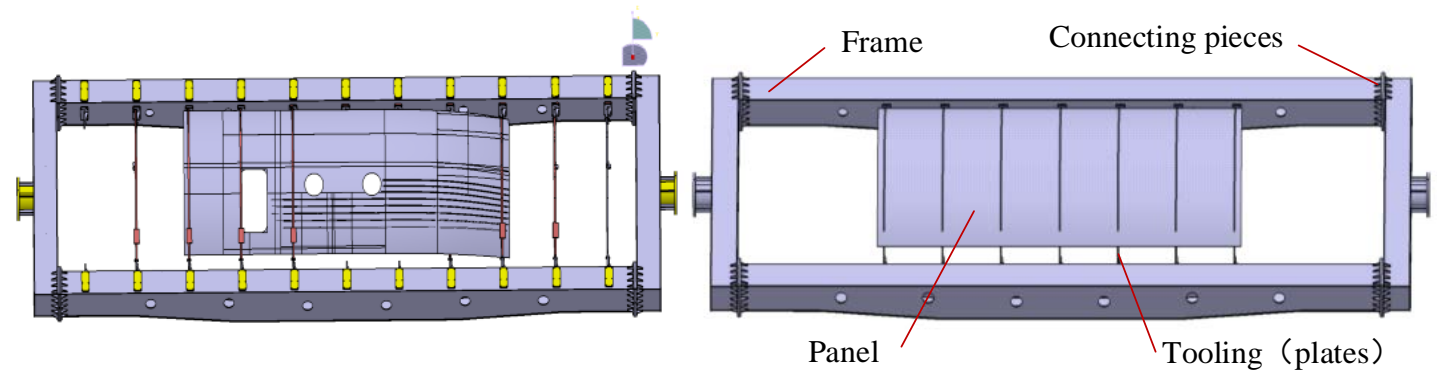

Fig. 2 The structure of bracket and panel before(left) and after(right) simplification

Import the model files reconstructed in CATIA into FEM software ABAQUS and define the material properties as table 1 shows.

Table 1 The material properties used for modeling

\begin{tabular}{cc}
\hline Mass Density (Kg/m3) & $7.86 \mathrm{e} 3$ \\
Young's Modulus (MPa) & $2 \mathrm{e} 5$ \\
Poisson's Ratio & 0.266 \\
Yield Strength (MPa) & 250 \\
\hline
\end{tabular}

Create a frequency extraction analysis step in ABAQUS, set eigenvalues solvers as LANCZOS, the number of eigenvalues requested as 30 . The boundary conditions of the fixed end of frame are defined as: free to rotate about the $\mathrm{Z}$ direction, all other degrees of freedom are constrained; the boundary conditions of the floating end of frame are defined as: free to move along the $Y$ direction and rotate about the $\mathrm{Z}$ direction, other degrees of freedom are constrained. The frame, connecting pieces, pallets and panel are all only subjected to its own weight. Divide the frame, connecting pieces, supporting pallets and panel into hexahedral meshes, element type is C3D8R, result after meshing is shown in figure 3.

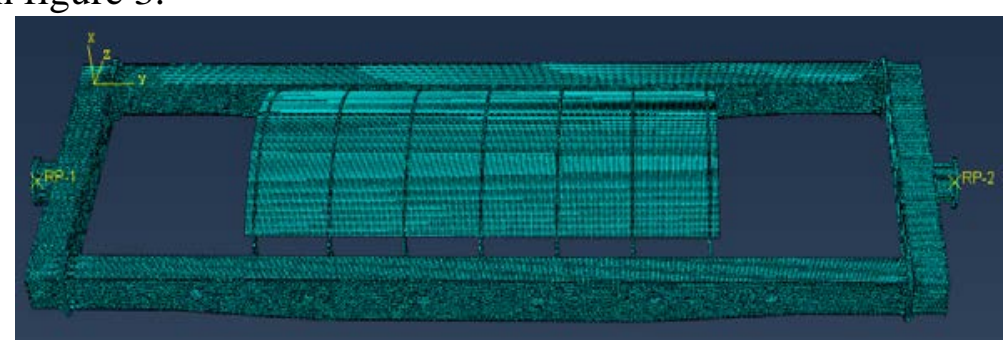

Fig. 3 The meshing diagram of bracket and panel 


\section{The modal analysis of bracket}

After submitting the analysis job in ABAQUS, the modal analysis of bracket is done, the natural frequencies and the corresponding inherent mode shapes are obtained. According to the existing dynamics study [9], we know that low order natural vibration effects larger on the vibration of the structure, so we usually take the former orders when doing analysis and calculation of structure vibration characteristics. The former 10 order modal analysis results of bracket are shown in table 2, the former 4 order modal shapes of bracket and product are shown in figure 4.

Table 2 The former 10 order modal analysis results of bracket

\begin{tabular}{ccc}
\hline Order & Frequency/Hz & Max displacement $/ \mathrm{mm}$ \\
\hline 1 & 9.596 & 1.015 \\
2 & 12.425 & 1.001 \\
3 & 13.333 & 1.004 \\
4 & 15.420 & 1.025 \\
5 & 16.653 & 1.262 \\
6 & 17.552 & 1.015 \\
7 & 18.936 & 1.001 \\
8 & 19.284 & 1.002 \\
9 & 19.706 & 1.002 \\
10 & 19.926 & 1.002 \\
\hline
\end{tabular}

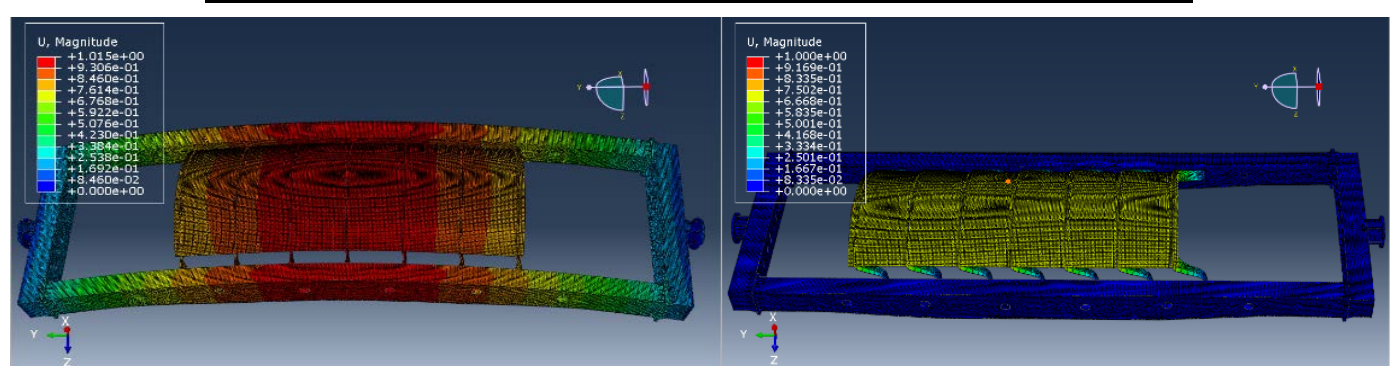

(a) The first-order modal shape

(b) The second-order modal shape

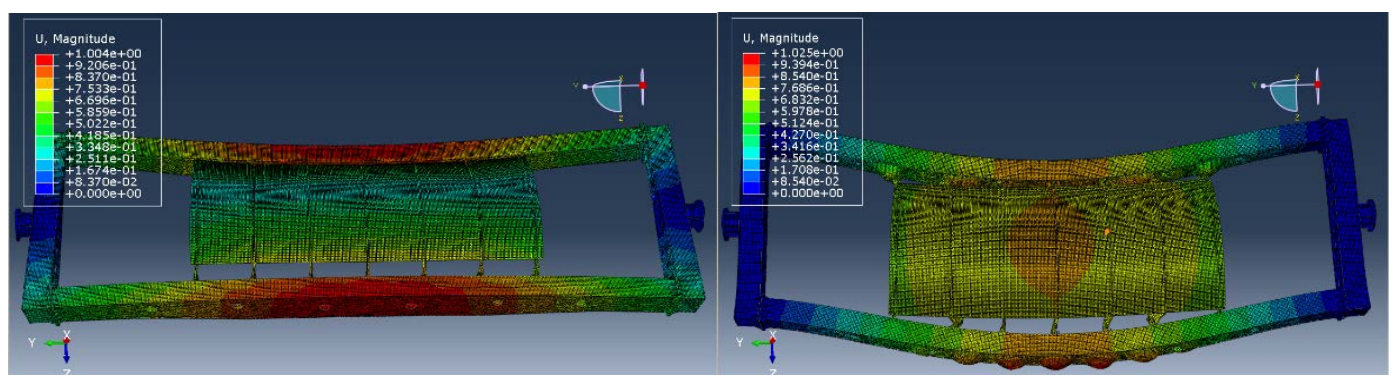

(c) The third-order modal shape

(d) The fourth-order modal shape

Fig. 4 The former 4 order modal shapes of bracket and panel

When the natural frequency of the system is equal to or close to the excitation frequency, there will be a resonance phenomenon, in this case the amplitudes and velocity amplitudes that under excitation increase with time. It can be seen from the constraint modal analysis of the structure that the former six order frequencies concentrated between $9.5 \mathrm{~Hz}$ and $17.5 \mathrm{~Hz}$, therefore, the excitation frequency should avoid this frequency range. Take the former four order modal shapes into a whole consideration, obviously the modal shape of the central part in cross beam has the largest relative displacement, so the cross beam should have a higher rigidity requirements and stronger vibration resistance.

Based on the above analysis, we can draw the following conclusions: 
(1) The low and medium frequency modal of the bracket structure are mainly bending vibration, so the external excitation frequencies should avoid the frequency range from $9.5 \mathrm{~Hz}$ to $17.5 \mathrm{~Hz}$;

(2) The high frequency modal of bracket structure belongs to the partial vibration modal, mainly focuses on the cross beam of the frame;

(3) The central section of frame and plates are the main parts of the vibration, so those part require a high vibration resistance;

(4) The global deformation of the bracket structure presents symmetry, which is determined by its structure symmetry.

\section{Summary}

The stability and reliability of bracket structure in automatic drill-riveting system make effects to the quality of aircraft panel. On the basis of illustrating the theory of modal analysis, this paper made a modal analysis for bracket structure based on ABAQUS, obtain the stress and displacement contours with the natural frequencies and its mode shapes under deadweight. It can be used to predict the bracket vibration performance without using samples test at the design stage. The result shows that this method for determining the vibration characteristics of the bracket structure works, it can be used for better structural designing to obtain higher vibration resistance, which has big significance to improve aircraft assembly accuracy.

Acknowledgement. This work is supported by National Natural Science Foundation of China (Grant No. 51375396).

\section{References}

[1] Yang Y, Wang Z Q, Kang Y G, et al. A Deformation Analysis and Compensation Algorithm for Bracket Structure of Automatic Drill-Riveting System[C]//Advanced Materials Research. 2014, 912: 539-544.

[2] Zeng Y, Dalvit D A R, O'Hara J, et al. Modal analysis method to describe weak nonlinear effects in metamaterials[J]. Physical Review B, 2012, 85(12): 125107.

[3] Kerschen G, Peeters M, Golinval J C, et al. Nonlinear modal analysis of a full-scale aircraft (AIAA)[J]. Journal of Aircraft, 2013, 50(5):1409-1419.

[4] Zhong B B, Zhang L, Tang Y H. Modal analysis of the automotive air compressor bracket[J]. Machine Building \& Automation, 2013.

[5] Patil S. Modal analysis of engine-mounting bracket using FEA for circular cross section[J]. Trends in Mechanical Engineering \& Technology, 2013, 3(1):23-28.

[6] Lu L, Qin Y, Cai Y. Modal analysis of rolling-sliding compound bearing based on ABAQUS[J]. Journal of Mechanical Transmission, 2014.

[7] Manohar C S. Applied Structural and Mechanical Vibrations - Theory and Methods, 2nd [J]. Journal of Sound \& Vibration, 2015, 336:293-295.

[8] Rainieri C, Fabbrocino G. Operational modal analysis of civil engineering structures : an introduction and guide for applications[M]. Springer, 2014.

[9] Park G, Sausse M, Inman D J, et al. Vibration Testing and Finite Element Analysis of an Inflatable Structure[J]. Aiaa Journal, 2012, 41(8):1556-1563. 